\title{
Detectability of Excitatory versus Inhibitory Drive in an Integrate-and-Fire-or-Burst Thalamocortical Relay Neuron Model
}

\author{
Gregory D. Smith ${ }^{1}$ and S. Murray Sherman ${ }^{2}$ \\ ${ }^{1}$ Department of Applied Science, College of William and Mary, Williamsburg, Virginia 23187, and 2Department of \\ Neurobiology, State University of New York, Stony Brook, New York 11794-5230
}

Although inhibitory inputs are often viewed as equal but opposite to excitatory inputs, excitatory inputs may alter the firing of postsynaptic cells more effectively than inhibitory inputs. This is because spike cancellation produced by an inhibitory input requires coincidence in time, whereas an excitatory input can add spikes with less temporal constraint. To test for such potential differences, especially in the context of the function of thalamocortical (TC) relay nuclei, we used a stochastic "integrate-and-fire-or-burst" TC neuron model to quantify the detectability of excitatory and inhibitory drive in the presence and absence of the low-threshold $\mathrm{Ca}^{2+}$ current, $I_{\mathrm{T}}$, and the hyperpolarization-activated cation conductance, $I_{\text {sag }}$. We find

Inhibitory inputs are often viewed as equal but opposite to excitatory inputs. That is, a reduction of postsynaptic firing resulting from an inhibitory input is often implied to convey information as effectively as an increase of postsynaptic firing caused by an excitatory input. For instance, the inhibitory GABAergic input from the basal ganglia to the ventral lateral and ventral anterior nuclei of the thalamus is often viewed as providing information to be relayed to the cortex (Purves et al., 1997; Kandel et al., 2000), just as excitatory input from the retina to the lateral geniculate nucleus or medial lemniscus to the ventrobasal complex is relayed to the cortex (Sherman and Guillery, 1996; Sherman and Koch, 1998). Other examples of inhibitory input include the cerebellar Purkinje cell projection to the deep cerebellar nuclei, inputs from the caudate nucleus to the substantia nigra pars reticulata, the substantia nigra to the superior colliculus, and many others.

However, inhibitory inputs are not equal to but in fact are opposite to excitatory ones. EPSPs affect the firing of the postsynaptic cell by adding action potentials, whereas IPSPs remove them. As long as the postsynaptic background firing rate is not near its upper limit, excitatory inputs can always add action potentials. However, because an inhibitory input can only cancel action potentials that are temporally coincident, it follows that IPSPs are ineffective at low postsynaptic firing rates.

Here we use a computational approach to test the hypothesis that excitatory and inhibitory inputs are not equally detectable

Received Aug. 1, 2002; revised Sept. 6, 2002; accepted Sept. 10, 2002.

This work was supported in part by National Science Foundation (NSF) Integrative Biology and Neuroscience Grant IBN 0228273 (G.D.S.) and National Eye Institute Grant EY03038 (S.M.S.). The work was performed in part using computational facilities at the College of William and Mary enabled by grants from the NSF and Sun Microsystems. We thank Hans E. Plesser for a careful reading of this manuscript.

Correspondence should be addressed to S. M. Sherman, Department of Neurobiology, State University of New York, Stony Brook, NY 11794-5230. E-mail: s.sherman@sunysb.edu.

Copyright (C) 2002 Society for Neuroscience 0270-6474/02/2210242-09\$15.00/0 that excitatory inputs are generally superior drivers compared with inhibitory inputs in part because spontaneous activity of a postsynaptic neuron is not required in the case of excitatory drive. Interestingly, the presence of the low-threshold $\mathrm{Ca}^{2+}$ current, $I_{\mathrm{T}}$ in a postsynaptic neuron allows the robust detection of inhibitory drive over a certain range of spontaneous and driven activity, a range that can be extended by the presence of the hyperpolarization-activated cation conductance, I sag. These simulations suggest a possible reinterpretation of the role of inhibitory inputs, such as those to the thalamus.

Key words: thalamus; inhibition; excitation; basal ganglia; neuron model; driver; modulator with regard to postsynaptic effectiveness. Because the thalamus is seen as a site for the relay of information and has both excitatory and inhibitory inputs believed to be the source of information (i.e., drivers as opposed to modulators) of thalamic relay nuclei (Sherman and Guillery, 1998), we use a thalamocortical (TC) relay neuron model to quantify the effect of voltage-dependent conductances, such as the low-threshold $\mathrm{Ca}^{2+}$ current, $I_{\mathrm{T}}$, and the hyperpolarization-activated cation conductance, $I_{\text {sag }}$, on the relative efficacy of excitatory versus inhibitory inputs.

Some of these results have been published previously in abstract form (Smith and Sherman, 2001).

\section{MATERIALS AND METHODS}

The integrate-and-fire-or-burst model. For a complete description of the development of the integrate-and-fire-or-burst (IFB) model and parameter selection, see Smith et al. (2000). Briefly, the IFB model is constructed by adding a slow variable to a classical integrate-and-fire neuron model (Rinzel, 1980; Keener et al., 1981; Tuckwell, 1988, 1989). Our simulations of the IFB model involve numerically integrating the following equations:

$$
\begin{gathered}
C \frac{d V}{d t}=-I_{\mathrm{L}}-I_{\mathrm{T}}-I_{\mathrm{S}}-I_{\mathrm{D}} \\
\frac{d h}{d t}=\left\{\begin{array}{cc}
-h / \tau_{\mathrm{h}}^{-} & \left(V \geq V_{\mathrm{h}}\right) \\
(1-h) / \tau_{\mathrm{h}}^{+} & \left(V<V_{\mathrm{h}}\right)
\end{array}\right.
\end{gathered}
$$

The current balance equation, Equation 1, includes a constant conductance leakage current $\left(I_{\mathrm{L}}\right)$ of the form, $I_{\mathrm{L}}=g_{\mathrm{L}}\left(V-V_{\mathrm{L}}\right)$; the lowthreshold $\mathrm{Ca}^{2+}$ current, $I_{\mathrm{T}}$; and two synaptic currents, $I_{\mathrm{S}}$ and $I_{\mathrm{D}}$. An action potential occurs whenever the membrane potential reaches the firing threshold $\left(V_{\theta}\right)$. After each action potential, an absolute refractory period of $t_{\mathrm{R}}=4 \mathrm{msec}$ is enforced during which the current balance equation, Equation 1, is not integrated and $V=V_{\text {reset }}$.

The low-threshold $\mathrm{Ca}^{2+}$ current takes the form $I_{\mathrm{T}}=g_{\mathrm{T}} m_{\infty} h\left(V-V_{\mathrm{T}}\right)$, where $m_{\infty}=\mu\left(V-V_{\mathrm{h}}\right)$ represents instantaneous voltage-dependent activation, and $\mu$ is the Heaviside step function. Equation 2 is an idealization of the dynamics of inactivation and deinactivation of $I_{\mathrm{T}}$ (Jahnsen and Llinas 1984a,b; Smith et al., 2000). 


\begin{tabular}{lll}
\hline \multicolumn{2}{l}{ Table 1. Parameters for the integrate-and-fire-or-burst } & model \\
Parameter & Value & Unit \\
\hline$V_{\theta}$ & -45 & $\mathrm{mV}$ \\
$V_{\mathrm{L}}$ & -65 & $\mathrm{mV}$ \\
$C$ & 2 & $\mu \mathrm{F} / \mathrm{cm}^{2}$ \\
$g_{\mathrm{L}}$ & 0.035 & $\mathrm{mS} / \mathrm{cm}^{2}$ \\
$V_{\text {reset }}$ & -50 & $\mathrm{mV}$ \\
$t_{\mathrm{R}}$ & 4 & $\mathrm{msec}$ \\
$V_{\mathrm{h}}$ & -60 (TRN like) & $\mathrm{mV}$ \\
& -70 (TC like) & \\
$V_{\mathrm{T}}$ & 120 & $\mathrm{mV}$ \\
$\tau_{\mathrm{h}}^{-}$ & 20 & $\mathrm{msec}$ \\
$\tau_{\mathrm{h}}^{+}$ & 100 & $\mathrm{msec}$ \\
$g_{\mathrm{T}}$ & 0.2 & $\mathrm{mS} / \mathrm{cm}^{2}$ \\
$V_{\text {sag }}$ & 0 & $\mathrm{mV}$ \\
$\tau_{\mathrm{r}}^{-}$ & 40 & $\mathrm{msec}$ \\
$\tau_{\mathrm{r}}^{+}$ & 200 & $\mathrm{msec}$ \\
$g_{\text {sag }}$ & 0.02 & $\mathrm{mS} / \mathrm{cm}^{2}$ \\
$V_{\text {spont }}$ & $0($ always excitatory) & $\mathrm{mV}$ \\
$V_{\mathrm{drive}}$ & -100 (inhibitory case) & $\mathrm{mV}$ \\
& $0($ excitatory case) & \\
$A_{\mathrm{S}}$ & 0.15 & $\mathrm{~ms}-\mathrm{mS} / \mathrm{cm}^{2}$ \\
$A_{\mathrm{D}}$ & 0.75 & $\mathrm{~ms}-\mathrm{mS} / \mathrm{cm}^{2}$ \\
$\tau$ & 1 & $\mathrm{msec}$ \\
\hline & & \\
\hline & &
\end{tabular}

TC-like and thalamic reticular-like simulations. Because of similarities between neurons of the thalamic reticular nucleus (TRN) and TC neurons, a subtle change in $V_{\mathrm{h}}$, the threshold for $I_{\mathrm{T}}$, or $V_{\mathrm{L}}$, the resting membrane potential, converts an IFB TC neuron model into a model that responds like TRN neurons. In TC cells at rest, $I_{\mathrm{T}}$ is inactivated (i.e., $V_{\mathrm{L}}>V_{\mathrm{h}}$ ), whereas TRN cells at rest are primed to burst in response to depolarizing input $\left(V_{\mathrm{L}}<V_{\mathrm{h}}\right)$. The phrases "TC-like" and "TRN-like" below refer to this tuning of parameters. In both cases, $V_{\mathrm{L}}=-65 \mathrm{mV}$ (Table 1). Some simulations included the hyperpolarization-activated cation current, $I_{\text {sag }}$, also known as $I_{\mathrm{h}}$. Parameters were chosen so that $I_{\text {sag }}$ causes the IFB model to burst rhythmically for a range of inhibitory applied current, consistent with experiment and detailed models of the intrinsic $0.5-4 \mathrm{~Hz}$ (delta) oscillation of cat TC neurons (Dossi et al., 1992; Wang, 1994).

Synaptic input. The last two terms in Equation $1, I_{\mathrm{S}}$ and $I_{\mathrm{D}}$, are synaptic currents attributable to excitatory spontaneous input $\left(I_{\mathrm{S}}\right)$ and excitatory or inhibitory drive $\left(I_{\mathrm{D}}\right)$. Each synaptic potential received by the IFB model neuron is modeled as an $\alpha$ function (Rall, 1967) of conductance with specified amplitude $\left(A_{\mathrm{S}}\right.$ or $\left.A_{\mathrm{D}}\right)$ and rise time $(\tau)$. For example, in the case of spontaneous input, an individual EPSP occurring at $t=0$ would be given by:

$$
g_{\mathrm{S}}(t)=\frac{A_{\mathrm{S}}}{\tau^{2}} t e^{-\mathrm{t} / \tau}(t \geq 0)
$$

where $\tau=1 \mathrm{msec}, A_{\mathrm{S}}=0.15 \mathrm{msec} \times \mathrm{mS} / \mathrm{cm}^{2}$, and the depolarizing postsynaptic current is $I_{\mathrm{S}}=g_{\mathrm{S}}\left(V-V_{\mathrm{S}}\right)$, with an excitatory reversal potential of $V_{\mathrm{S}}=0 \mathrm{mV}$. Spontaneous EPSP event times were modeled as a Poisson process with rate $\rho_{\mathrm{S}}$, and the resulting synaptic input was generated using the method put forth by Destexhe et al. (1994). The EPSPs or IPSPs that are the result of driven input were modeled similarly $\left(V_{\mathrm{D}}=0\right.$ or $-100 \mathrm{mV} ; A_{\mathrm{D}}=5 A_{\mathrm{S}}=0.75 \mathrm{msec} \times \mathrm{mS} / \mathrm{cm}^{2}$; rate $\left.\rho_{\mathrm{D}}\right)$.

We integrated the equations for the IFB model using a $1 \mathrm{GHz}$ LIN UX workstation running XPP, an ordinary differential equation solver written by Bard Ermentrout at the University of Pittsburgh (Pittsburgh, PA) (http://www.pitt.edu/ phase/). All calculations were performed using the fourth-order Runge-Kutta integration method and a time step of $10-50 \mu \mathrm{sec}$.

Receiver operator characteristic analysis of simulated responses. Receiver operator characteristic (ROC) analysis (Green and Swets, 1966; MacMillan and Creelman, 1991) of IFB model response was performed using a modified MATLAB 6 (The MathWorks) subroutine written by Fabrizio
A

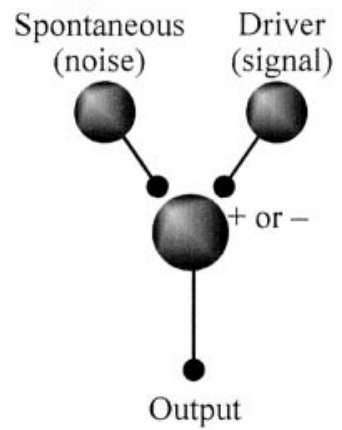

B

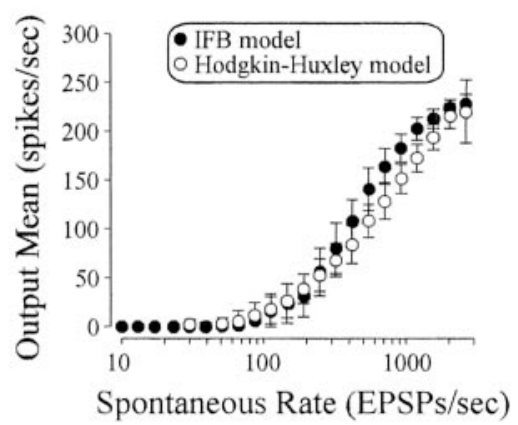

Figure 1. A, Diagram of the configuration of the model neuron that is postsynaptic to two sources (input). The first input [Spontaneous (noise)] is excitatory, and if sufficiently intense, it causes a certain level of background activity in the postsynaptic neuron. The second input [Driver (signal)] represents either excitatory or inhibitory drive, the presence or absence of which must be detected based on spike count (Output). B, Filled circles, output rate of the IFB model (mean $\pm \mathrm{SD}$ ) as a function of the rate of spontaneous EPSPs. Open circles, Mean output rate of the Hodgkin-Huxley style thalamocortical relay neuron model. Parameters are as described in Table 1 and Materials and Methods.

Gabbiani at Baylor College of Medicine (Houston, TX) (Gabbiani and Koch, 1998) (see http://glab.bcm.tmc.edu/). For given rates of spontaneous $\left(\rho_{\mathrm{S}}\right)$ and driven $\left(\rho_{\mathrm{D}}\right)$ activity (Fig. $\left.1 A\right)$, multiple simulations were performed to produce realizations of two random variables, $S$ and $D$, that represent the number of spikes occurring in a window of specified duration (usually $t=50$ or $200 \mathrm{msec}$ ). Although the random variable $S$ represents the spike count caused by spontaneous excitatory input at rate $\rho_{\mathrm{S}}$, the random variable $D$ represents spike count caused by a combination of this spontaneous input and either excitatory or inhibitory drive at rate $\rho_{\mathrm{D}}$. Each round of ROC analysis involves ranging over $28 \times 28$ (i.e., 784) pairs of the rates $\rho_{\mathrm{S}}$ and $\rho_{\mathrm{D}}$ and for each pair of rates simulating 100-1000 neural responses. These results served as a numerical estimate of the probability mass functions of $S$ and $D$ from which ROC area was calculated (Guido et al., 1995). ROC area parameter studies were performed on SciClone, a Beowulf-like parallel computing system at the College of William and Mary composed of several clusters of networked workstations from Sun Microsystems (http://www.compsci.wm.edu/sciclone).

See http://www.as.wm.edu/Faculty/Smith.html for an extended description of the above methods.

\section{RESULTS \\ ROC calculations without the low-threshold calcium current}

To quantify the detectability of excitatory versus inhibitory drive for a model of a thalamic neuron responding purely in tonic mode, we used the IFB model with the conductance underlying $I_{\mathrm{T}}$ (i.e., $g_{\mathrm{T}}$ ) set to zero. Thus, Equation 2 is irrelevant, and the IFB model behaves as a classical integrate-and-fire model that receives EPSPs or IPSPs from two sources (Fig. 1 $A$ ). The first source provides EPSPs at rate $\rho_{\mathrm{S}}$, and this spontaneous excitation (i.e., noise) is supplemented by input at rate $\rho_{\mathrm{D}}$ from a second source that represents either excitatory or inhibitory drive (i.e., signal).

The focus of this work is the extent to which the presence or absence of the driver input is detectable in the output of the postsynaptic neuron (Fig. $1 A$ ). Because this may depend on spontaneous activity, Figure $1 B$ shows the output rate (mean \pm $\mathrm{SD}$ ) of the IFB model as a function of the spontaneous EPSP rate. The input-output relationship increases monotonically and saturates at 250 spikes/sec, because the absolute refractory period of the model, $t_{\mathrm{R}}$, is $4 \mathrm{msec}$. Saturation becomes appreciable only 


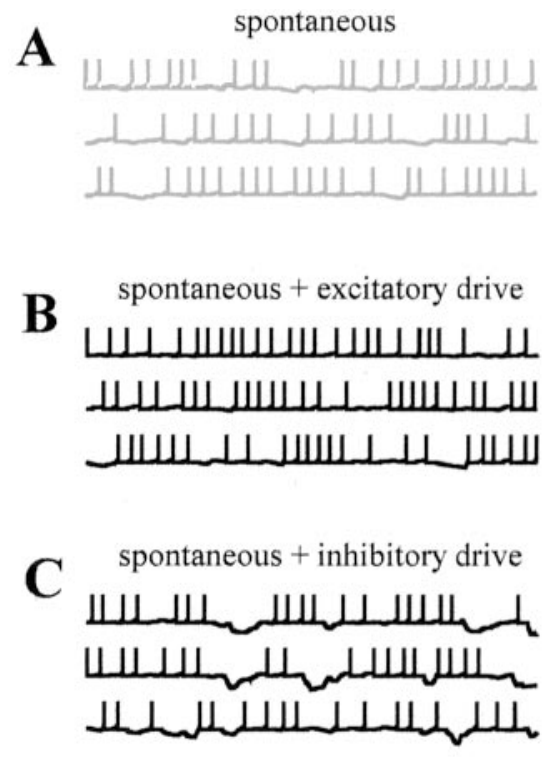

Figure 2. A, Representative membrane potential time courses $(200 \mathrm{msec}$ in duration) for an integrate-and-fire model receiving spontaneous EPSPs at 400 EPSPs/ sec. $B$, Time courses when $400 \mathrm{EPSPs} / \mathrm{sec}$ of spontaneous input is augmented by 50 EPSPs/sec excitatory drive. $C$, Time courses when $400 \mathrm{EPSPs} / \mathrm{sec}$ of spontaneous input is attenuated by 50 IPSPs/sec inhibitory drive. $D$, Histogram of spike counts for 1000 trials (from $A$ and $B$ ) and a diagram representing the corresponding ROC area calculation (see Materials and Methods), where the probability (Pr) of a hit is plotted against the probability of a false alarm. $E$, Histogram of spike counts for 1000 trials (from $A$ and $C$ ).
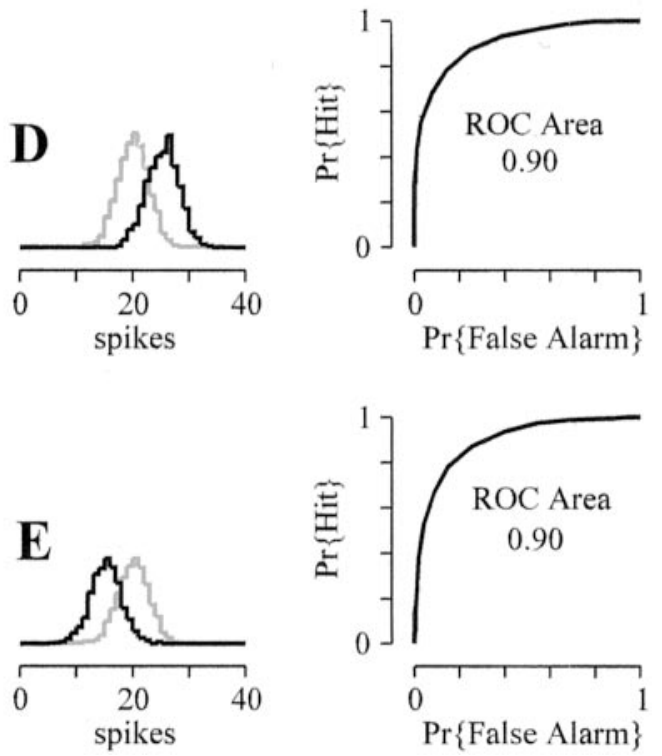

when the spontaneous input rate is greater than approximately $\rho_{\mathrm{S}}=50 \mathrm{EPSPs} / \mathrm{sec}$, because several EPSPs must summate to evoke a spike in the postsynaptic neuron.

Figure $2 A$ shows three representative $200 \mathrm{msec}$ plots of the IFB model membrane potential for a spontaneous input rate of $\rho_{\mathrm{S}}=$ $400 \mathrm{EPSPs} / \mathrm{sec}$. The number of output spikes varies generally between 10 and 30 . The histogram of Figure $2 D$ summarizes the response of this neuron in the absence of drive (mean \pm SD of $98 \pm 13$ spikes/sec). In Figure $2 B$, excitatory drive is $\rho_{\mathrm{D}}=50$ EPSPs/sec, and the histogram of spike counts shifts rightward $(124 \pm 14.4$ spikes/sec). In Figure $2 D$, a ROC area calculation (see Materials and Methods) quantifies the detectability of this excitatory drive. For $\rho_{\mathrm{S}}=400 \mathrm{EPSPs} / \mathrm{sec}$ and $\rho_{\mathrm{D}}=50 \mathrm{EPSPs} / \mathrm{sec}$, the ROC area is 0.9 , i.e., very detectable $(0.5$ is the chance level, and 1 represents perfect detectability). Figure 2, $C$ and $E$, shows that when the postsynaptic neuron receives an inhibitory drive at 50 IPSPs/sec, the response of the postsynaptic neuron is suppressed (5-25 spikes), and the spike count histogram shifts leftward, giving a ROC area of 0.9 .

Thus, for a neuron receiving $400 \mathrm{EPSPs} / \mathrm{sec}$, the detectability of 50 additional EPSPs/sec and 50 IPSPs/sec is comparable. However, this is not true in general and is, in fact, a consequence of the background activity of the postsynaptic neuron (98 spikes/ sec). To clarify the dependence of this result on the rate of spontaneous and driven input, Figure 3 presents a summary of ROC area calculations performed for $28 \times 28$ (i.e., 784) values of $\rho_{\mathrm{S}}$ and $\rho_{\mathrm{D}}$. For each combination, 100 trials of $50 \mathrm{msec}$ duration were simulated to construct a histogram of spike count that corresponds to the "spontaneous plus excitatory drive" case (similar to Fig. $2 B$ and the black curve in Fig. 2D). Next, the ROC area was calculated by comparing these histograms to the distribution of spike count using identical spontaneous EPSP rate and zero excitatory drive (similar to Fig. $2 A$ and the gray curve in Fig. $2 D$ ).

Figure $3 A$ shows that for spontaneous input rates below $\sim 100$ EPSPs/sec, a relatively constant amount of excitatory drive leads to detectable signal (for a ROC area of 0.8, 15 EPSPs/sec drive is required). However, when the spontaneous input rate increases to $>100$ EPSPs/sec, the amount of excitatory drive necessary to maintain detectability increases. This leads to a sloped (nonvertical) interface between low and high ROC area for a $\rho_{\mathrm{S}}$ of $>100$
EPSPs/sec. Despite this changing threshold of detectability, ROC area is always a monotonic increasing function of the rate of excitatory drive (left to right). Thus, elevated spontaneous input can suppress the detectability of excitatory inputs, but increasing excitatory drive never decreases detectability (Fig. $3 A$ ).

Figure 3, $A$ and $D$, shows that excitatory and inhibitory drivers are not equivalent with respect to postsynaptic detectability. In the inhibitory case, spontaneous activity in the postsynaptic neuron is required so that inhibitory drive may be detected as a suppressed spike count (i.e., detectability is near zero for a $\rho_{\mathrm{S}}$ of $<100$ EPSPs/sec). To clarify this point, Figure 4, $A$ and $C$, shows the ROC analysis of Figure 3, $A$ and $D$, replotted with the mean output rate of the postsynaptic neuron in the absence of drive on the $y$-axis (no change to the $x$-axis, which still represents the rate of excitatory or inhibitory drive). This transformation of the $y$-axis is possible because in the absence of drive, the mean firing rate of the postsynaptic neuron is a monotonic increasing function of the rate of spontaneous EPSPs (recall Fig. 1B). Figure $4 B$ shows that for a ROC area of 0.8 , spontaneous activity of $\sim 30$ EPSPs/sec is needed. However, there is no such requirement in the case of excitatory drive (Fig. $4 A$ ).

\section{ROC calculations with a TC-like, low-threshold calcium current}

To quantify the detectability of excitatory versus inhibitory drive in a TC neuron model that includes the low-threshold $\mathrm{Ca}^{2+}$ current, $I_{\mathrm{T}}$, the parameter $g_{\mathrm{T}}$ in the IFB model was changed to the standard value of $0.2 \mathrm{mS} / \mathrm{cm}^{2}$ (Table 1). Here $V_{\mathrm{h}}$, the threshold for activation/inactivation of $I_{\mathrm{T}}$, is $-70 \mathrm{mV}, 5 \mathrm{mV}$ less than the resting membrane potential, $V_{\mathrm{L}}=-65 \mathrm{mV}$. Thus, $I_{\mathrm{T}}$ is TC-like (i.e., inactivated at rest), and the IFB model responds with bursts only after release from hyperpolarization.

Figure 3, $B$ and $E$, summarizes ROC analysis for the TC-like IFB model under the influence of spontaneous EPSPs and either excitatory (Fig. $3 B$ ) or inhibitory (Fig. $3 E$ ) drive. Figure $3 B$ is nearly identical to Figure $3 A$, so the presence of $I_{\mathrm{T}}$ has little effect on the detectability of excitatory drive, because all inputs to the IFB model are depolarizing and, consequently, $I_{\mathrm{T}}$ remains inactivated. However, Figure $3, D$ and $E$, shows that $I_{\mathrm{T}}$ has a significant effect on the detectability of inhibitory drive. In this case, 


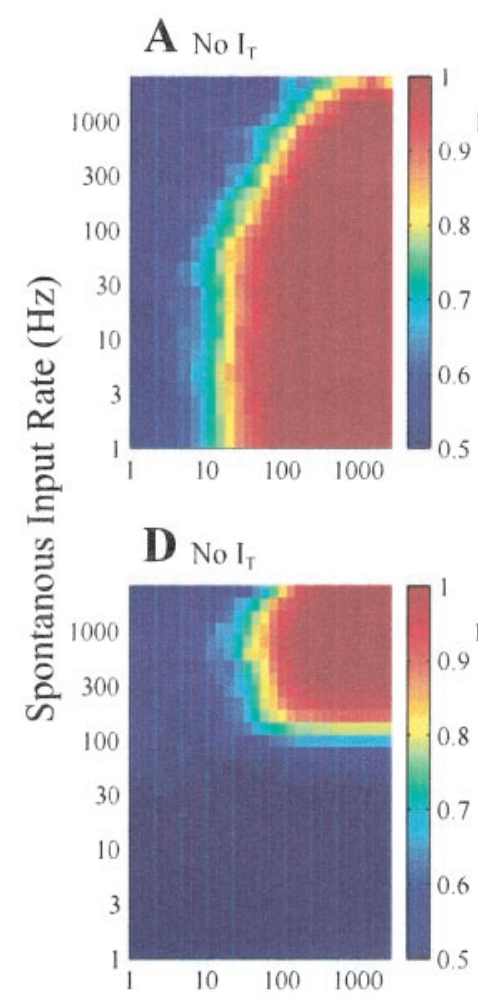

B TC-like

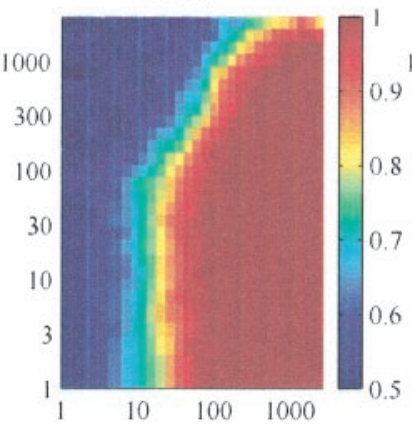

E TC-like

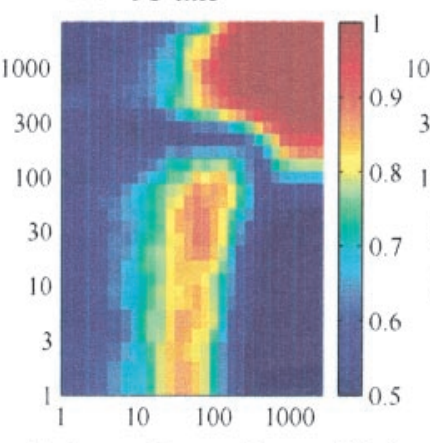

Driven Input Rate $(\mathrm{Hz})$
C TRN-like

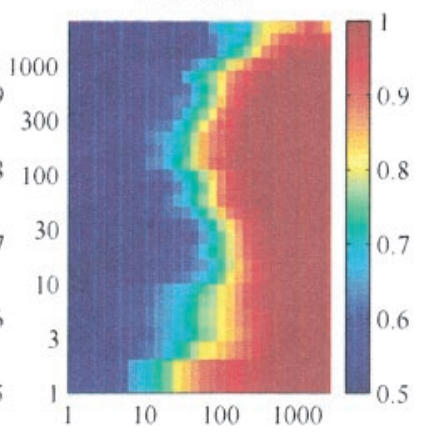

F TRN-like $^{2}$

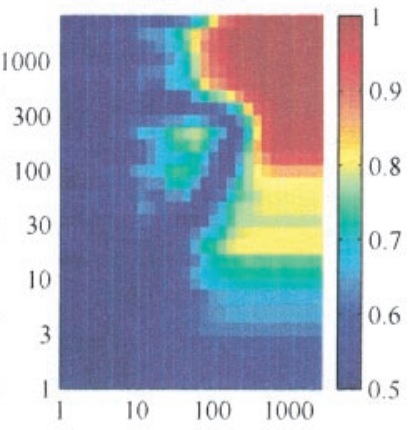

Figure 3. Summary of ROC area calculations performed for various combinations of spontaneous EPSP rate (spontaneous input rate; $y$-axis) and rate of excitatory or inhibitory drive (driven input rate; $x$-axis). ROC area plots summarize $\sim 800$ combinations of spontaneous input rate and driven input rate, each leading to a number between 0.5 (chance level) and 1 (perfectly detectable). $A$, ROC area when the low-threshold $\mathrm{Ca}^{2+}$ current, $I_{\mathrm{T}}$, is not included in the simulations $\left(g_{\mathrm{T}}=0.2 \mathrm{mS} / \mathrm{cm}^{2}\right) . B, I_{\mathrm{T}}$ is included in the model in a TC-like manner $\left(g_{\mathrm{T}}=0.2 \mathrm{mS} / \mathrm{cm}^{2} ; V_{\mathrm{h}}=-70 \mathrm{mV} ; V_{\mathrm{L}}=\right.$ $-65 \mathrm{mV}) . C, I_{\mathrm{T}}$ is included in the model in a TRN-like manner $\left(g_{\mathrm{T}}=0.2 \mathrm{mS} / \mathrm{cm}^{2}\right.$; $\left.V_{\mathrm{h}}=-60 \mathrm{mV} ; V_{\mathrm{L}}=-65 \mathrm{mV}\right) . D-F$, Same as $A-C$, except the drive is inhibitory.

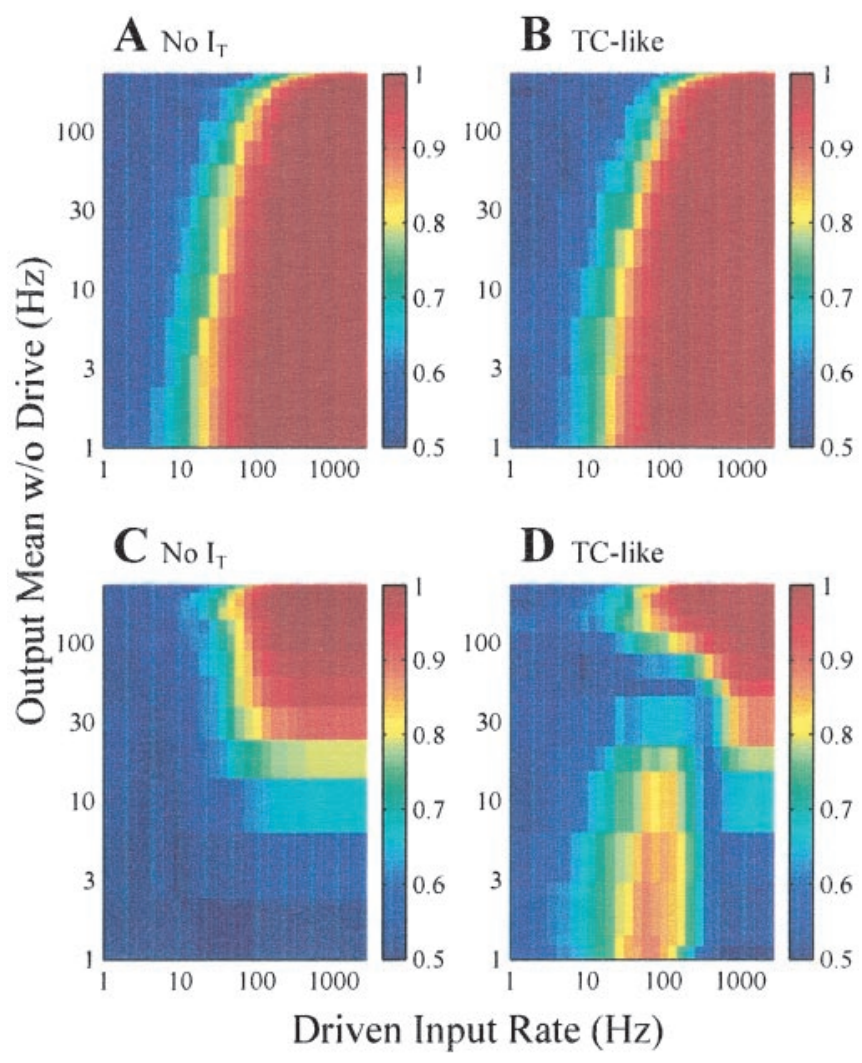

Figure 4. ROC analysis from Figure $3, A, B, D$, and $E$ replotted as a function of mean output rate of the postsynaptic neuron in the absence of drive ( $y$-axis) and rate of excitatory or inhibitory drive ( $x$-axis). This transformation is possible because in the absence of drive, the mean firing rate of the postsynaptic neuron is a monotonic increasing function of the spontaneous EPSP rate. clusters of IPSPs from the inhibitory drive occasionally deinactivate $I_{\mathrm{T}}$. If additional inhibition does not occur, then the membrane potential relaxes toward $V_{\mathrm{L}}$ until the burst threshold $V_{\mathrm{h}}$ is crossed, thereby activating $I_{\mathrm{T}}$. Often an appropriately timed spontaneous EPSP accelerates this process. The elevated ROC area observed in Figure $3 E\left(\rho_{\mathrm{S}}<160\right.$ EPSPs/sec and two IPSPs/sec $<$ $\rho_{\mathrm{D}}<100$ IPSPs/sec) is attributable to bursts that lead to an elevated spike count distinguishable from that observed in the absence of inhibitory drive; other regions of high ROC area in Figure $3, D$ and $E$, reflect a reduced spike count. Figure $4 D$ also shows that in the presence of $I_{\mathrm{T}}$, spontaneous activity is not required for inhibitory drive to be detectable. Notice that the location of elevated ROC area implies that detectability is no longer a monotonic increasing function of the rate of inhibitory drive; that is, if the inhibitory drive is sufficiently strong, the relief from inhibition required for bursts does not occur.

To clarify this, Figure 5 shows three representative plots of the IFB model membrane potential for a spontaneous (excitatory) input rate of $\rho_{\mathrm{S}}=30 \mathrm{EPSPs} / \mathrm{sec}$ (Fig. $5 A$ ). Consistent with Figure $1 B$, this rate of spontaneous input leads to almost no response (Fig. 5D, gray line) (less than one trial in a thousand results in a spike). However, when this spontaneous input is supplemented with an excitatory drive rate of $\rho_{\mathrm{D}}=30 \mathrm{EPSP} / \mathrm{sec}$ in Figure $5 B$, tonic spikes are observed, and the histogram shifts rightward (28.4 \pm 10.8 spikes/sec) (Fig. 5D, black line).

In Figure $5 C$, inhibitory drive is included at a rate of $\rho_{\mathrm{D}}=30$ IPSPs/sec, resulting in numerous IPSPs. Interestingly, although the driving input is inhibitory, the histogram of spike counts again shifts rightward $(29.1 \pm 14.7$ spikes/sec $)$. Focusing on the ROC area in the case of inhibitory drive, we see that postinhibitory rebound bursting mediated by $I_{\mathrm{T}}$ causes the inhibitory drive to be nearly perfectly detectable $(0.99$ in Fig. $5 E$ ), despite the fact 
Figure 5. A, Representative membrane potential time courses $(200 \mathrm{msec}$ in duration) for the IFB model with TC-like, low-threshold $\mathrm{Ca}^{2+}$ current receiving spontaneous EPSPs at $30 \mathrm{EPSP} / \mathrm{sec}$ (no response). $B$, Time courses when 30 EPSPs/sec of spontaneous input is augmented by 30 IPSPs/sec of excitatory drive. $C$, Cell response increases when 30 EPSPs/sec of spontaneous input is combined with 30 IPSPs/sec of inhibitory drive. $D, E$, Histograms of spike counts for 1000 trials and the corresponding ROC area calculations. $\mathrm{Pr}$, Probability.

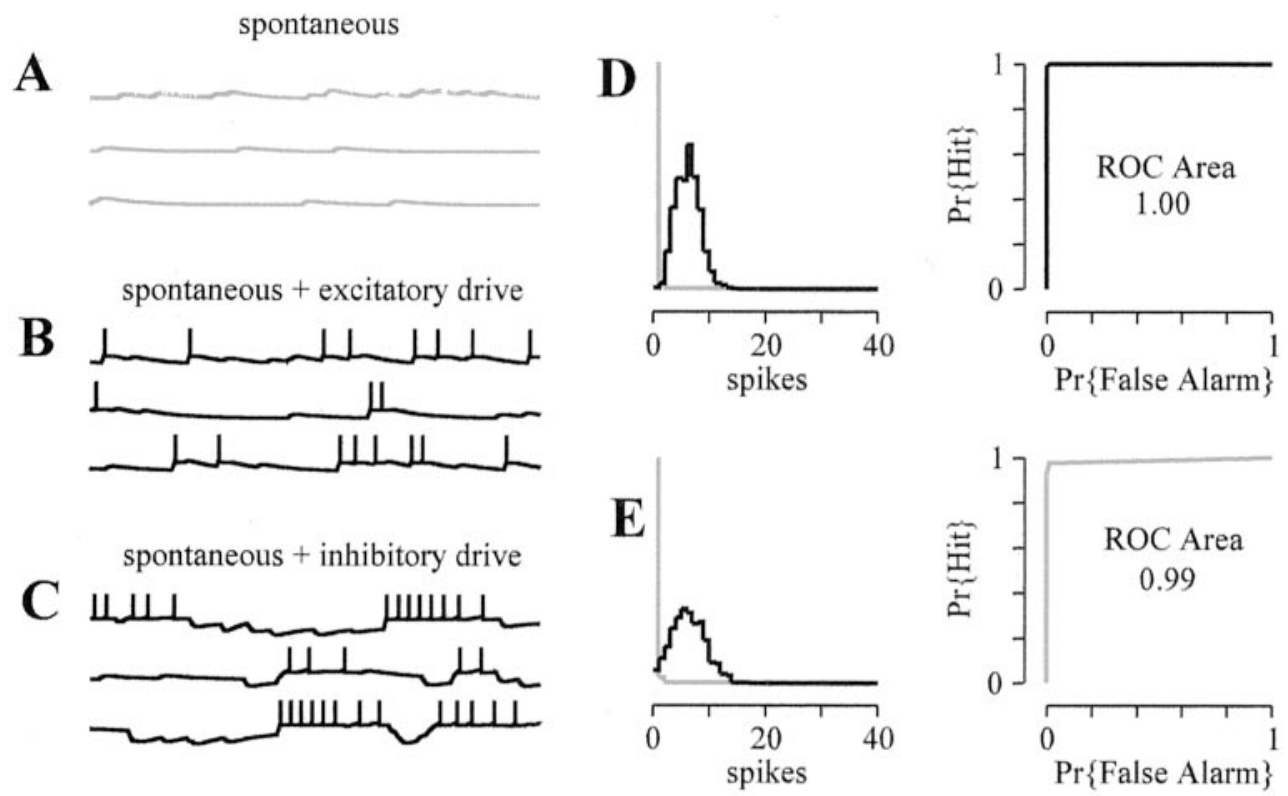

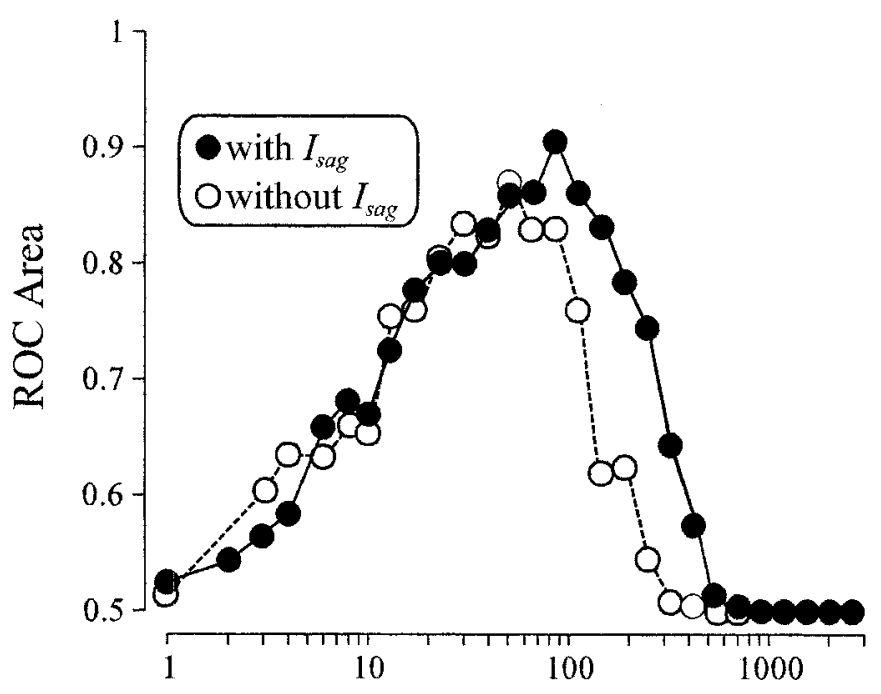

\section{Driven IPSP Rate (spikes/sec)}

Figure 6. ROC analysis for the IFB model in the presence $(\bigcirc)$ and absence $(\bullet)$ of the hyperpolarization-activated cation current, $I_{\text {sag }}$, as a function of the rate of inhibitory drive ( $x$-axis) for a spontaneous excitatory input rate of $10 \mathrm{EPSPs} / \mathrm{sec}$. In the absence of $I_{\mathrm{sag}}$, this corresponds to a cross section of Figure $3 E$.

that the spontaneous activity of the neuron is negligible in the absence of drive (Fig. 5A).

When the IFB model neuron was augmented to include the hyperpolarization-activated cation current, $I_{\text {sag }}$, ROC area calculations were qualitatively similar to those performed in the absence of this low-threshold current for both excitatory and inhibitory drive (data not shown; results are similar to Fig. 3B,E). Interestingly, $I_{\text {sag }}$ does enlarge the region over which the presence of $I_{\mathrm{T}}$ allows the detection of inhibitory drive (Fig. 6).

\section{ROC calculations with a TRN-like, low-threshold calcium current}

In TC cells at rest, $I_{\mathrm{T}}$ is inactivated, i.e., the resting membrane potential is greater than the threshold for activation/inactivation of the low-threshold $\mathrm{Ca}^{2+}$ current $\left(V_{\mathrm{L}}>V_{\mathrm{h}}\right)$. However, in TRN neurons, the threshold for activation of $I_{\mathrm{T}}$ is more depolarized than that in TC cells (Huguenard and Prince, 1992). Thus, we set the parameter $V_{\mathrm{h}}$ to $-60 \mathrm{mV}(5 \mathrm{mV}$ greater than the resting membrane potential, $V_{\mathrm{L}}=-65 \mathrm{mV}$ ) so that the IFB model is TRN-like $\left(V_{\mathrm{h}}>V_{\mathrm{L}}\right)$ and responds to sufficiently intense depolarization with a low-threshold $\mathrm{Ca}^{2+}$ spike.

Figure 3, $C$ and $F$, summarizes ROC analysis for the TRN-like IFB model when the drive is either excitatory (Fig. $3 C$ ) or inhibitory (Fig. $3 F$ ). Although the overall comparison of $C$ and $F$ with $B$ and $E$ in Figure 3 gives the impression that the effects of TRN-like $I_{\mathrm{T}}$ are subtle, there are some differences. For example, the distinction between Figure 3, $C$ and $A$, is certainly greater than that between Figure $3, B$ and $A$, demonstrating that the presence of $I_{\mathrm{T}}$ influences the detectability of excitatory drive in the TRN-like IFB model (as opposed to the TC-like case, in which it had no effect). Interestingly, the presence of TRN-like $I_{\mathrm{T}}$ has an overall effect of decreasing the region of elevated ROC area. In particular, the threshold for detectability of excitatory inputs increased when the spontaneous input rate was 10-100 EPSPs/sec (see notch in Fig. 3, $C$ compared with $A$ ).

The ROC areas in the presence of TRN-like $I_{\mathrm{T}}$ and inhibitory drive (Fig. $3 F$ ) are distinct from both the TC-like case (Fig. 3E) and the result in the absence of $I_{\mathrm{T}}$ (Fig. 3D). There is an island of detectability for spontaneous input rates between 30 and 300 EPSPs/sec. At $\rho_{\mathrm{S}}=100 \mathrm{EPSP} / \mathrm{sec}$, for example, ROC area first increases, then decreases, and then increases again as a function of the rate of inhibitory drive $\left(\rho_{\mathrm{S}}\right)$. The elevated detectability for spontaneous input rates between 30 and $300 \mathrm{EPSPs} / \mathrm{sec}$ is analogous to the region of elevated ROC area in the TC-like case. Here, the excitatory spontaneous input depolarizes the model membrane potential enough so that certain levels of inhibitory drive lead to postinhibitory rebound bursts. This is demonstrated in Figure $7 C$ using $\rho_{\mathrm{S}}=100$ EPSPs/sec and $\rho_{\mathrm{D}}=30$ IPSPs $/ \mathrm{sec}$. The TRN-like IFB model shows increased spike count for excitatory drive (Fig. $7 B$ ), but for these values of $\rho_{\mathrm{S}}$ and $\rho_{\mathrm{D}}$, the detectability of inhibitory drive $(0.94)$ (Fig. $7 E$ ) is greater than that of excitatory drive (0.82) (Fig. $7 D)$.

Figure $3 F$ shows moderate ROC area $(0.7-0.8)$ extending to 

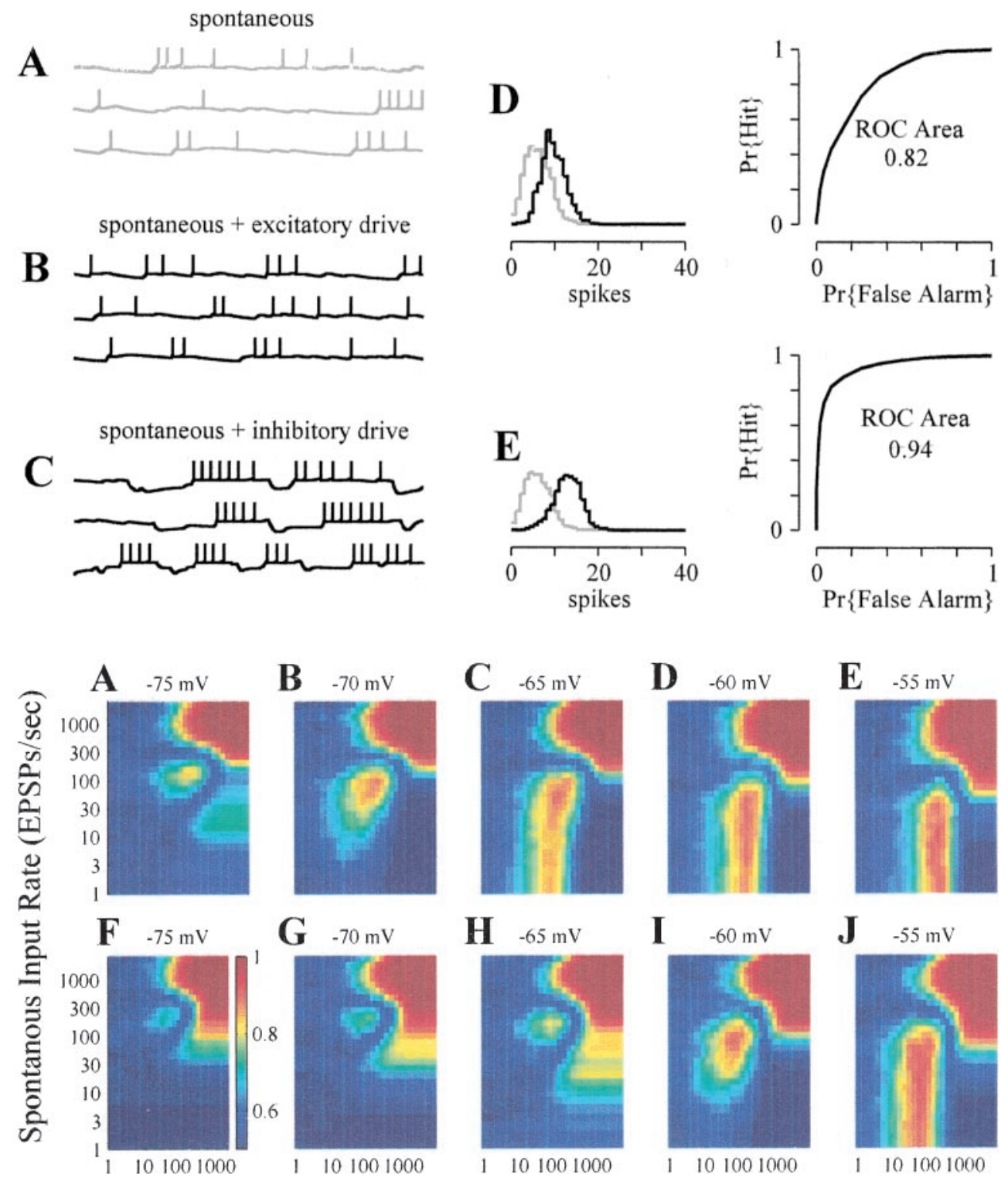

Driven Input Rate (IPSPs/sec)
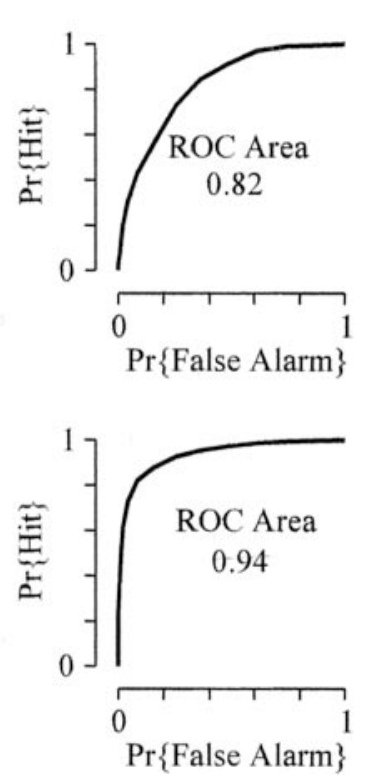

Figure 7. $A$, Representative membrane potential time courses for the IFB model with TRN-like, low-threshold $\mathrm{Ca}^{2+}$ current receiving spontaneous EPSPs at 100 EPSPs/sec. B, Time courses when 30 EPSPs/sec of excitatory drive is included. $C$, Cell response increases when $30 \mathrm{IP}$ $\mathrm{SPs} / \mathrm{sec}$ of inhibitory drive is included. $D$, $E$, Histograms of spike counts for 1000 trials and the corresponding ROC area calculations. Pr, Probability.
Figure 8. ROC area calculations resulting from inhibitory drive, where $V_{\mathrm{L}}$ is varied from -75 to $-55 \mathrm{mV} . A-E, V_{\mathrm{h}}=$ $-70 \mathrm{mV}$ so that $C$ is a reproduction of the TC-like result of Figure $3 E$, where the resting membrane potential is given by the standard value of $V_{\mathrm{L}}=-65 \mathrm{mV} . F-J$, $V_{\mathrm{h}}=-60 \mathrm{mV}$ so that $H$ is a reproduction of the TRN-like result of Figure $3 F$. lower levels of spontaneous EPSP rates than in the absence of $I_{\mathrm{T}}$ (compare Fig. $3 D$ ), although the effect is not as strong as when $I_{\mathrm{T}}$ is TC-like (compare Fig. $3 E$ ) and occurs over a different range of rates of inhibitory drive (Fig. $3 F, \rho_{\mathrm{D}}$ of $>100$ IPSPs/sec; Fig. $3 E$, $\rho_{\mathrm{D}}$ of $<100$ IPSPs/sec). This region of moderate ROC area is attributable to detectable suppression of bursts that are induced by spontaneous excitatory input in the absence of inhibitory drive (data not shown).

\section{Voltage dependence of ROC calculations}

In the above simulations, ROC area analysis has been performed using the IFB model both with and without $I_{\mathrm{T}}$. In the presence of $I_{\mathrm{T}}$, we have found that the detectability of inhibitory inputs was dependent on the relationship between the IFB model resting membrane potential, $V_{\mathrm{L}}$, and the threshold $\left(V_{\mathrm{h}}\right)$ for activation/ inactivation of $I_{\mathrm{T}}$. In particular, a region of elevated detectability of inhibitory inputs that is prominent when the IFB model is TC-like and $I_{\mathrm{T}}$ is inactivated at rest $\left(V_{\mathrm{L}}>V_{\mathrm{h}}\right)$ (Fig. $3 E$ ) is reduced or absent when the IFB model is TRN-like and $I_{\mathrm{T}}$ is deinactivated at rest $\left(V_{\mathrm{L}}<V_{\mathrm{h}}\right)($ Fig. $3 F)$. However, because modulatory inputs can change the resting membrane potential of both TC and TRN neurons, it is important to clarify how the detectability of inhibitory input may depend on the resting membrane potential.

To address this question for inhibitory inputs, Figure $8 A-E$ presents ROC area calculations in which $V_{\mathrm{L}}$ was varied from -75 through $-55 \mathrm{mV}$ (Table 1). In these calculations, $V_{\mathrm{h}}=-70 \mathrm{mV}$, and Figure $8 C$ thus reproduces Figure $3 E$. Figure $8, D$ and $E$, shows that changing $V_{\mathrm{L}}$ to more depolarized values $(-60$ or -55 $\mathrm{mV}$ ) has little effect on the ROC area calculation. That is, Figure $8 C-E$ shows a region of elevated detectability for inhibitory inputs induced by $I_{\mathrm{T}}$. Conversely, Figure $8, A$ and $B$, shows that changing the resting membrane potential to more hyperpolarized values $\left(V_{\mathrm{L}}=-75\right.$ or $\left.-70 \mathrm{mV}\right)$ reduces the detectability of inhibitory inputs. Interestingly, the result in Figure $8 A$ (where $V_{\mathrm{L}}=-75$ and $V_{\mathrm{h}}=-70 \mathrm{mV}$ ) is very similar to the ROC analysis of the TRN-like IFB model shown in Figure $3 F$ (where $V_{\mathrm{L}}=-65$ and $\left.V_{\mathrm{h}}=-60 \mathrm{mV}\right)$, indicating the importance of the relationship 
between $V_{\mathrm{L}}$ and $V_{\mathrm{h}}$ in determining whether the model bursts in response to depolarization or relief from hyperpolarization and the consequent detectability (or lack thereof) of inhibitory input. Indeed, the overall structure of Figure $8 A-J$ (where $V_{\mathrm{h}}=-60 \mathrm{mV}$ so that Fig. $8 H$ thus corresponds to the TRN-like result of Fig. $3 F$ ) suggests that the important features of the ROC analysis plots are determined by the quantity $V_{\mathrm{L}}-V_{\mathrm{h}}$.

Calculations similar to those presented in Figure 8 indicate that the resting membrane potential affects the detectability of excitatory input as well (data not shown). However, the similarity of TC-like and TRN-like responses to excitatory inputs (Fig. 3, compare $B$ with $C$ ) means that the variation in the ROC analysis is modest compared with that observed in Figure 8.

\section{ROC area analysis using a Hodgkin-Huxley-like, conductance-based model}

To determine the degree to which the above results generalize, we performed ROC analysis using a truncation of the McCormick-Huguenard TC neuron model (Huguenard and McCormick, 1992, 1994; McCormick and Huguenard, 1992; Mukherjee and Kaplan, 1995). The current balance equation included five currents: fast $\mathrm{Na}^{+}$and $\mathrm{K}^{+}$(delayed rectifier) currents responsible for action potential generation, $I_{\mathrm{Na}}$ and $I_{\mathrm{K}-\mathrm{DR}} ; \mathrm{K}^{+}$and $\mathrm{Na}^{+}$leak currents, $I_{\text {Kleak }}$ and $I_{\text {Naleak }}$; the low-threshold $\mathrm{Ca}^{2+}$ current, $I_{\mathrm{T}}$; and in some cases, $I_{\text {sag }}$ (for details, see http://www.as.wm.edu/ Faculty/Smith.html). Action potentials were counted according to the number of times the membrane potential was depolarized above an arbitrary threshold value of $-30 \mathrm{mV}$. We found the ROC area analysis of the Hodgkin-Huxley (HH) and IFB models to be in quantitative agreement, regardless of whether the drive was excitatory or inhibitory. This agreement is attributable in part to the similarity of the input-output relationships of the $\mathrm{HH}$ and IFB models (data not shown) when both are constrained to fit experimental observations of TC neuron responses from cat thalamic slices (Smith et al., 2001).

\section{ROC calculations with balanced excitatory and inhibitory spontaneous input}

In the simulations presented thus far, the detectability of excitatory or inhibitory drive has been quantified assuming that the background activity of the IFB model is caused solely by excitatory spontaneous input. However, it is more realistic to presume that the background activity of the output neuron in Figure $1 A$ is caused by a mixture of excitatory and inhibitory spontaneous input. To represent this possibility, the current balance equation for the IFB model was extended to include two spontaneous synaptic current terms, one excitatory and the other inhibitory. When the spontaneous excitatory and inhibitory inputs are balanced (both arriving at identical $\rho_{\mathrm{S}}$ rates), the input-output relationship for the IFB model in the absence of drive is significantly shallower than the result for purely excitatory spontaneous input (data not shown).

Despite this difference, the ROC analysis for balanced excitatory and inhibitory spontaneous input is qualitatively similar to the results for pure excitatory spontaneous input (data not shown, similar to Fig. 3). However, in both the absence and the presence of $I_{\mathrm{T}}$, the combination of excitatory and inhibitory spontaneous input does slightly improve detectability of excitatory input when the spontaneous input rate is high ( $\rho_{\mathrm{S}}$ of $>100$ PSPs/sec). When the driving input is inhibitory, the combination of excitatory and inhibitory spontaneous input tends to decrease detectability at high spontaneous input rates but does not eliminate the region of elevated ROC area observed in the presence of $I_{\mathrm{T}}$ (compare Fig. $3 E$ ). When the parameters for $I_{\mathrm{T}}$ are TRN-like, a corresponding trend is observed; mixed excitatory and inhibitory spontaneous input leads to a slight increase in detectability when the drive is excitatory and a slight decrease in detectability when the drive is inhibitory, but only when the spontaneous input rate is high.

\section{DISCUSSION}

Using an integrate-and-fire-or-burst TC neuron model and a more detailed Hodgkin-Huxley-style model (data not shown), we have quantified the detectability of stochastic excitatory and inhibitory drive in the presence and absence of the low-threshold $\mathrm{Ca}^{2+}$ current, $I_{\mathrm{T}}$. By calculating ROC area, we quantified detectability when the model neuron received postsynaptic potentials from an excitatory source that evokes spontaneous activity (i.e., noise) and a second (excitatory or inhibitory) source that represents an afferent signal (Fig. $1 A$ ). Similar results were obtained when the model neuron received spontaneous postsynaptic potentials from a combination of excitatory and inhibitory sources (data not shown).

We find that in the absence of $I_{\mathrm{T}}$ detectability is typically poorer for inhibitory than for excitatory drive. This is particularly true for low to moderate levels of spontaneous activity in the postsynaptic neuron, because spontaneous activity is required for inhibitory drive to be detected as a suppressed spike count. In the presence of $I_{\mathrm{T}}$, results depend strongly on whether the threshold for $I_{\mathrm{T}}$ activation lies above the resting membrane potential (TRNlike) or below it (TC-like). In the latter case, $I_{\mathrm{T}}$ enables the detection of inhibitory drivers even when the postsynaptic neuron is not spontaneously active.

The qualitative aspects of the ROC area analysis presented here are not sensitive to the size of the spontaneous and driven postsynaptic potentials ( $A_{\mathrm{S}}$ and $A_{\mathrm{D}}$ ), although a threefold change does shift areas of elevated detectability toward higher or lower EPSP/IPSP rates (data not shown). Interestingly, we found that changes in resting membrane potential can significantly change the overall dependence of the detectability of inhibitory input on spontaneous and driven input rates (Fig. 8). This suggests that the detection of inhibitory inputs enabled by $I_{\mathrm{T}}$ may be sensitive to neuromodulation that affects the resting membrane potential of TC neurons.

\section{Limitations of single-compartment modeling}

Because both of the models used (IFB and Hodgkin-Huxley) are single-compartmental models, a limitation of this work is that some results may not generalize to situations involving temporally or spatially patterned synaptic input distributed over dendritic arbors. Although excitatory and inhibitory inputs are demonstrably not equivalent in isopotential compartmental models, one might devise a multicompartmental model with various assumptions about dendritic architecture, the distribution of ionic currents, and the spatial organization of synaptic inputs, for which excitatory and inhibitory inputs are less distinguishable on the basis of detectability. Nevertheless, the use of single-compartmental models is appropriate on several grounds. As a practical matter, the choice is motivated by a desire to perform adequate statistics of the stochastic responses that we quantify. Furthermore, previous cable modeling of current flow within dendritic arbors of thalamic relay cells has concluded that they are electronically compact. Thus, a postsynaptic potential generated anywhere in the dendritic arbor of a relay cell spreads with relatively little attenuation throughout the arbor and to its soma (Bloomfield and Sherman, 1989). Finally, 
the experimental evidence that would allow one to associate various compartments of a multicompartmental model with specific synaptic inputs suggests that excitatory and inhibitory inputs are largely overlapping (Wilson et al., 1984). In the absence of spatial heterogeneity of excitatory and inhibitory inputs, we expect the results of multicompartmental modeling to largely agree with the single compartment modeling results presented here.

\section{Can inhibitory inputs be drivers to thalamus?}

It has been stressed previously (Sherman and Guillery, 1998, 2001) that afferent inputs to thalamic relay cells can be divided into at least two functionally distinct groups: drivers and modulators. Drivers of thalamic relay cells transmit the basic information to be relayed to the cortex, act through ionotropic receptors that have a fast postsynaptic effect, and, where practical, have been identified as the transmitter of receptive field properties. Modulators, on the other hand, may activate metabotropic receptors having a slow and prolonged postsynaptic effect, and these afferents produce only subtle changes in receptive field properties. The retinal input to the lateral geniculate nucleus, the inferior collicular input to the medial geniculate nucleus, and the medial lemniscal input to the ventrobasal complex are examples of drivers, and so are cortical layer 5 inputs to many higher-order thalamic relays. Although drivers can be clearly recognized for some other thalamic relays, the identity of the drivers is not yet obvious in some nuclei (Sherman and Guillery, 1998, 2001). Examples of modulators include local GABAergic inputs, corticothalamic feedback from layer 6 and cholinergic, noradrenergic, and serotonergic inputs from brainstem. It is possible to view the spontaneous (noise) input leading to background activity in the IFB model as a modulator, because the time constant for spontaneous EPSPs can be slowed considerably without qualitative changes to the detectability analysis (data not shown).

A number of criteria to distinguish drivers from modulators for thalamic relays have been suggested; for example, crosscorrelograms from driver inputs are likely to be sharply peaked compared with those from modulatory inputs. Certainly an important functional criteria for a driver input is an ability to transfer information efficiently across the synapse to the relay cell, and thus, the presence or absence of driving input must be detectable on the basis of the postsynaptic neuron response. In the absence of knowledge about presynaptic firing rates, our simulations show that overall detectability is poorer for inhibitory drive than excitatory drive. Thus, we suggest adding to the list of criteria to distinguish drivers from modulators the proposition that, other things being equal, inhibitory inputs are less likely to be drivers than excitatory inputs.

However, an important caveat to this conclusion is that over a certain range of spontaneous and driven activity, the lowthreshold $\mathrm{Ca}^{2+}$ current, $I_{\mathrm{T}}$, may allow the detection of inhibitory drive. Indeed, the calculations of elevated detectability mediated by $I_{\mathrm{T}}$ presented above suggest three criteria for the identification of an inhibitory driver that uses $I_{\mathrm{T}}$ : (1) a candidate inhibitory driver must provide IPSPs at a rate that results in postinhibitory rebound bursting and elevated spike count in the postsynaptic neuron (in Fig. 3E, this range is $10-100 \mathrm{IPSPs} / \mathrm{sec}$ ), (2) the spontaneous activity of the postsynaptic neuron must be modest ( $<30$ spikes/sec in Fig. 4D), and (3) for robust detectability, the postsynaptic neuron must express the low-threshold $\mathrm{Ca}^{2+}$ current in a TC-like manner, so that inhibitory drive can deinactivate $I_{\mathrm{T}}$. In light of these results, we suggest that inhibitory projections to thalamic relay nuclei should not be presumed to be drivers unless the spontaneous activity of the postsynaptic neuron is relatively high or there is reason to believe that these three conditions for $I_{\mathrm{T}}$-mediated detectability hold. Because relay cells responding in burst mode have nonlinear input-output relationships, we also suggest that $I_{\mathrm{T}}$-mediated detection of inhibitory drive is unlikely to be a mechanism associated with faithful relay of information to the cortex (Sherman, 2001).

The implications of these criteria for inhibitory drivers are potentially far reaching. For example, textbook accounts (Purves et al., 1997; Kandel et al., 2000) imply that the basal ganglia relays information to the neocortex via a GABAergic inhibitory input to ventral anterior and lateral nuclei of the thalamus. Because available evidence suggests that the responses of relay cells of these nuclei are primarily in tonic mode (Zirh et al., 1998; Radhakrishnan et al., 1999; Magnin et al., 2000), it is unlikely that these nuclei detect inhibitory input via an $I_{\mathrm{T}}$-dependent mechanism. Thus, using the criteria for inhibitory drivers listed above, one might suggest that these nuclei detect inhibition through the suppression of spontaneous and relatively high-frequency tonic responses. Alternatively, the basal ganglia input to the ventral anterior and lateral nuclei of the thalamus might not be functioning as an inhibitory driver but rather as an inhibitory modulator of another input or inputs to these thalamic nuclei, for example, input from the cerebellum (Mason et al., 2000; Sakai et al., 2000) and/or layer 5 of the motor cortex (cf. Guillery and Sherman, 2002).

To give another example, the identification of inhibitory Purkinje cells of the cerebellar cortex as drivers of the deep cerebellar nuclei may or may not be consistent with the criteria for inhibitory drivers discussed above. If not, another candidate driver input to the deep cerebellar nuclei is excitation from mossy or climbing fiber axon branches, in which case Purkinje cell activity would be an inhibitory modulator rather than an inhibitory driver, providing the basic information for the deep cerebellar nuclei to transmit to other brain centers. Rethinking of the functional organization of this and other neural pathways may be required if responses of presumed inhibitory drivers and their postsynaptic targets are not consistent with the criteria for inhibitory drivers proposed above.

\section{REFERENCES}

Bloomfield SA, Sherman SM (1989) Dendritic current flow in relay cells and interneurons of the cat's lateral geniculate nucleus. Proc Natl Acad Sci USA 86:3911-3914.

Destexhe A, Mainen Z, Sejnowski T (1994) Synthesis of models for excitable membranes, synaptic transmission and neuromodulation using a common kinetic formalism. J Comput Neurosci 1:195-230.

Dossi RC, Nunez A, Steriade M (1992) Electrophysiology of a slow $(0.5-4 \mathrm{~Hz})$ intrinsic oscillation of cat thalamocortical neurones in vivo. J Physiol (Lond) 447:215-234.

Gabbiani F, Koch C (1998) Signal processing techniques for spike train analysis using MatLab. In: Methods in neuronal modeling: from ions to networks, Ed 2 (Koch C, Segev I, eds). Cambridge, MA: MIT.

Green D, Swets J (1966) Signal detection theory and psychophysics. New York: Wiley.

Guido W, Lu S-M, Vaughan J, Godwin D, Sherman SM (1995) Receiver operating characteristic (ROC) analysis of neurons in the cat's lateral geniculate nucleus during tonic and burst response mode. Vis Neurosci 12:723-741.

Guillery RW, Sherman SM (2002) Thalamic relay functions and their role in corticocortical communication: generalizations from the visual system. Neuron 33:1-20.

Huguenard JR, McCormick D (1992) Simulation of the currents involved in rhythmic oscillations in thalamic relay neurons. J Neurophysiol 68:1373-1383.

Huguenard J, McCormick D (1994) Electrophysiology of the neuron. New York: Oxford UP.

Huguenard JR, Prince DA (1992) A novel T-type current underlies prolonged $\mathrm{Ca}^{2+}$-dependent burst firing in GABAergic neurons of rat thalamic reticular nucleus. J Neurosci 12:3804-3817. 
Jahnsen H, Llinas R (1984a) Electrophysiological properties of guineapig thalamic neurones: an in vitro study. J Physiol (Lond) 349:205-226.

Jahnsen H, Llinas R (1984b) Ionic basis for the electroresponsiveness and oscillatory properties of guinea-pig thalamic neurones in vitro. J Physiol (Lond) 349:227-247.

Kandel ER, Schwartz JH, Jessell TM (2000) Principles of neural science. New York: McGraw Hill.

Keener J, Hoppensteadt F, Rinzel J (1981) Integrate-and-fire models of nerve membrane response to oscillatory input. SIAM J Appl Math 41:503-517.

MacMillan N, Creelman C (1991) Detection theory: a user's guide. Cambridge, MA: Cambridge UP.

Magnin M, Morel A, Jeanmonod D (2000) Single-unit analysis of the pallidum, thalamus and subthalamic nucleus in Parkinsonian patients. Neuroscience 96:549-564.

Mason A, Ilinsky IA, Maldonado S, Kultas-Ilinsky K (2000) Thalamic terminal fields of individual axons from the ventral part of the dentate nucleus of the cerebellum in Macaca mulatta. J Comp Neurol 421:412-428.

McCormick D, Huguenard J (1992) A model of the electrophysiological properties of thalamocortical relay neurons. $J$ Neurophysiol 68:1384-1400.

Mukherjee P, Kaplan E (1995) Dynamics of neurons in the cat lateral geniculate nucleus: in vivo electrophysiology and computational modeling. J Neurophysiol 74:1222-1243.

Purves D, Augustine GJ, Fitzpatrick D, Katz LC, Lamantia A-S, McNamara JO (1997) Neuroscience. Sunderland, MA: Sinauer.

Radhakrishnan V, Tsoukatos J, Davis KD, Tasker RR, Lozano AM, Dostrovsky JO (1999) A comparison of the burst activity of lateral thalamic neurons in chronic pain and non-pain patients. Pain 80:567-575.

Rall W (1967) Distinguishing theoretical synaptic potentials computed for different soma-dendritic distributions of synaptic inputs. J Neurophysiol 30:1138-1168.

Rinzel J (1980) Models in neurobiology. In: Nonlinear phenomena in physics and biology, Vol 75 (Enns RH, Jones BL, Miura RM, Rangnekar SS, eds), pp 347-367. Alberta, Canada: North Atlantic Treaty Association Advanced Study Institute.
Sakai ST, Stepniewska I, Qi HX, Kaas JH (2000) Pallidal and cerebellar afferents to pre-supplementary motor area thalamocortical neurons in the owl monkey: a multiple labeling study. J Comp Neurol 417:164-180.

Sherman SM (2001) Tonic and burst firing: dual modes of thalamocortical relay. Trends Neurosci 24:122-126.

Sherman SM, Guillery RW (1996) The functional organization of thalamocortical relays. J Neurophysiol 76:1367-1395.

Sherman SM, Guillery RW (1998) On the actions that one nerve cell can have on another: distinguishing "drivers" from "modulators". Proc Natl Acad Sci USA 95:7121-7126.

Sherman SM, Guillery RW (2001) Exploring the thalamus. San Diego: Academic.

Sherman SM, Koch C (1998) Thalamus. In: Synaptic organization of the brain, Ed 4 (Shepherd G, ed), pp 246-278. New York: Oxford UP.

Smith G, Sherman SM (2001) Detectability of excitatory versus inhibitory drive in a stochastic thalamocortical relay neuron model. Soc Neurosci Abstr 27:723.21.

Smith G, Cox C, Sherman SM, Rinzel J (2000) Fourier analysis of sinusoidally-driven thalamocortical relay neurons and a minimal intergrate-and-fire-or-burst model. J Neurophysiol 83:588-610.

Smith G, Cox C, Sherman SM, Rinzel J (2001) Spike-frequency adaptation in sinusoidally-driven thalamocortical relay neurons. Thalamus and Related Systems 1:135-156.

Tuckwell H (1988) Introduction to theoretical neurobiology: nonlinear and stochastic theories, Vol 2. Cambridge, UK: Cambridge UP.

Tuckwell H (1989) Stochastic processes in the neurosciences. Philadelphia: Society for Industrial and Applied Mathematics.

Wang XJ (1994) Multiple dynamical modes of thalamic relay neurons: rhythmic bursting and intermittent phase-locking. Neuroscience 59:21-31.

Wilson JR, Friedlander MJ, Sherman SM (1984) Fine structural morphology of identified X- and Y-cells in the cat's lateral geniculate nucleus. Proc R Soc Lond B Biol Sci 221:411-436.

Zirh TA, Lenz FA, Reich SG, Dougherty PM (1998) Patterns of bursting occurring in thalamic cells during parkinsonian tremor. Neuroscience $83: 107-121$. 